\title{
Recent development in the strategies projected for chikungunya vaccine in humans
}

This article was published in the following Dove Press journal:

Drug Design, Development and Therapy

\author{
Manu Goyal' \\ Anil Chauhan ${ }^{2}$ \\ Vishavdeep Goyal ${ }^{3}$ \\ Nishant Jaiswal ${ }^{1,2}$ \\ Shreya Singh ${ }^{4}$ \\ Meenu Singh ${ }^{1,2}$ \\ 'Department of Pediatrics, \\ Postgraduate Institute of \\ Medical Education and Research, \\ Chandigarh, India; ${ }^{2}$ Indian Council \\ of Medical Research Advanced \\ Centre for Evidence Based Child \\ Health, Postgraduate Institute of \\ Medical Education and Research, \\ Chandigarh, India; ${ }^{3}$ Apollo Hospitals \\ International Limited, Ahmedabad, \\ India; ${ }^{4}$ Department of Medical \\ Microbiology, Postgraduate Institute \\ of Medical Education and Research, \\ Chandigarh, India
}

\begin{abstract}
The unprecedented epidemic spread of chikungunya worldwide illustrates the critical need for potent vaccines and therapeutic interventions. The morbidity and mortality associated with this arboviral infection has become a major public health problem in many countries across different continents. Increasing public-private partnerships have opened new avenues in research and development of vaccines. This review mainly focuses on the recent advances in patented approaches for chikungunya vaccine development and the forthcoming challenges.
\end{abstract}

Keywords: chikungunya, epidemics, CHIKV vaccine candidates, clinical trials, humans

\section{Introduction}

Chikungunya is an emerging arthritogenic arboviral illness caused by chikungunya virus (CHIKV), a member of the genus Alphavirus in the Togaviridae family. CHIKV is an enveloped, positive-sense, single-stranded RNA with $11.8 \mathrm{~kb}$ long linear genome with two open reading frames encoding four nonstructural replicase proteins (nsP1, nsP2, nsP3, and nsP4) and five structural proteins (three glycosylated envelope glycoproteins E1, E2, and E3, nonglycosylated nucleocapsid and cleavage product $6 \mathrm{k}$ ). CHIKV was first isolated during an epidemic in Tanganyika in 1953. In fact, the name "chikungunya" pronounced as "CHI-kən-GUUN-yə" is derived from the Kimakonde language of the Makonde tribe, which means "the bent walker/stooped walking" describing the contorted posture of a patient suffering with severe joint pain and other arthritic symptoms caused by this disease. ${ }^{1,2}$ The virus has evolved into three distinct geographical lineages with distinct genotypes - East/Central/South African (ECSA), Asian, and West African. ${ }^{3}$ Explosive outbreaks during the last decade worldwide have prompted National Institute of Allergy and Infectious Diseases (NIAID) to add CHIKV in the category C priority pathogen biodefense list (https://www.niaid.nih.gov/research/emerging-infectious-diseases-pathogens).

The virus transmission takes place by the arthropod vectors, female Aedes aegyptica or Aedes albopictus mosquitoes; aggressive daytime feeders that also transmit dengue, zika and yellow fever. ${ }^{2}$ The routine sylvatic or nonhuman cycle involves other primates like apes and lemurs but during epidemics, chikungunya transmission can be continued by interhuman spread. ${ }^{4} A$. albopictus - the Asian tiger mosquito - is a more coldtolerant mosquito and adaptation of a single amino-acid genetic mutation in the E1 glycoprotein of CHIKV strains resulted in the epidemiologic shift of chikungunya from tropical to temperate regions of the world. ${ }^{5}$

\section{Search strategy}

This review is based on all types of studies related to CHIKV vaccine development in humans and discussed the various vaccine approaches in detail. The articles 
were retrieved by PubMed, OVID, EMBASE, Google search, and clinical trials registries for relevant studies using search strategy (Search $(((()(($ chickungunya) OR chikungunya) OR ((Chikungunya Fever) OR Chikungunya virus $)))$ AND (((()((inactivated vaccine) OR live attenuated vaccine) $\mathrm{OR}$ virus like particle vaccine) $\mathrm{OR}$ recombinant vaccine) OR chikungunya virus vaccine)) OR ((((chikungunya virus vaccine) OR vaccine) OR conjugate vaccine) OR virus like protein $)))$ AND (((()(((clinical trials) OR random) OR randomly) OR phase 1) OR phase 2) OR phase 3) OR randomised controlled trial) OR randomized))) NOT ((animals) NOT humans)). The search included publications that are recent till August, 2018. We have searched for studies to be included irrespective of any language or publication status. We have also manually searched the references and bibliographies of all the included studies and related review articles for any additional studies. Figure 1 shows the flowchart for screening and study selection process.

\section{Epidemiology}

The dissemination of the CHIKV is geographically distributed in tropical and subtropical regions of East Africa, Southeast Asia, and Western Pacific. The persistent increase in unchecked vector population, immunologically naïve population, climatic changes, urbanization, and ease of global travel has elevated the risk for geographical expansion of CHIKV to nonendemic regions. The first outbreak of chikungunya was documented in late 1950 s to early 1960 s in Bangkok and from 1963 to 1973 in India, affecting thousands of people. The next two major outbreaks were reported in coastal regions of Kenya in 2004 and consequently spread to numerous islands of Indian Ocean over a period of next 4 years. The largest outbreak occurred in islands of La-Reunion, in the western part of Indian Ocean in 2005 in which 266,000 cases and 254 deaths were reported by French public health authorities. ${ }^{6}$ Several other countries including Mauritius, Seychelles, Madagascar, Gabon, Comoros and Mayotte, India, Sri Lanka, Indonesia, Thailand, Senegal, Philippines, Laos, Guinea, Taiwan, Vietnam, Myanmar, Singapore, Malaysia, Cambodia, and Pakistan also reported chikungunya cases. Massive chikungunya outbreaks were documented in India during 2006-2007, affecting $>1.5$ million people. ${ }^{7,8}$ The disease then spread rapidly toward Pacific islands causing significant outbreaks in the Caribbean Islands in $2011 .{ }^{9}$ Post the upsurge of Indian Ocean outbreak, the disease spread to a few places in Europe also. The CHIKV was later introduced in Americas in late 2013. The number of chikungunya cases spiraled during the devastating 2014-2015 epidemic in several North, Central, and South American countries resulting in millions of reported cases. ${ }^{10,11}$ This was one of the most prominently reported outbreaks of CHIKV in Americas. The series of

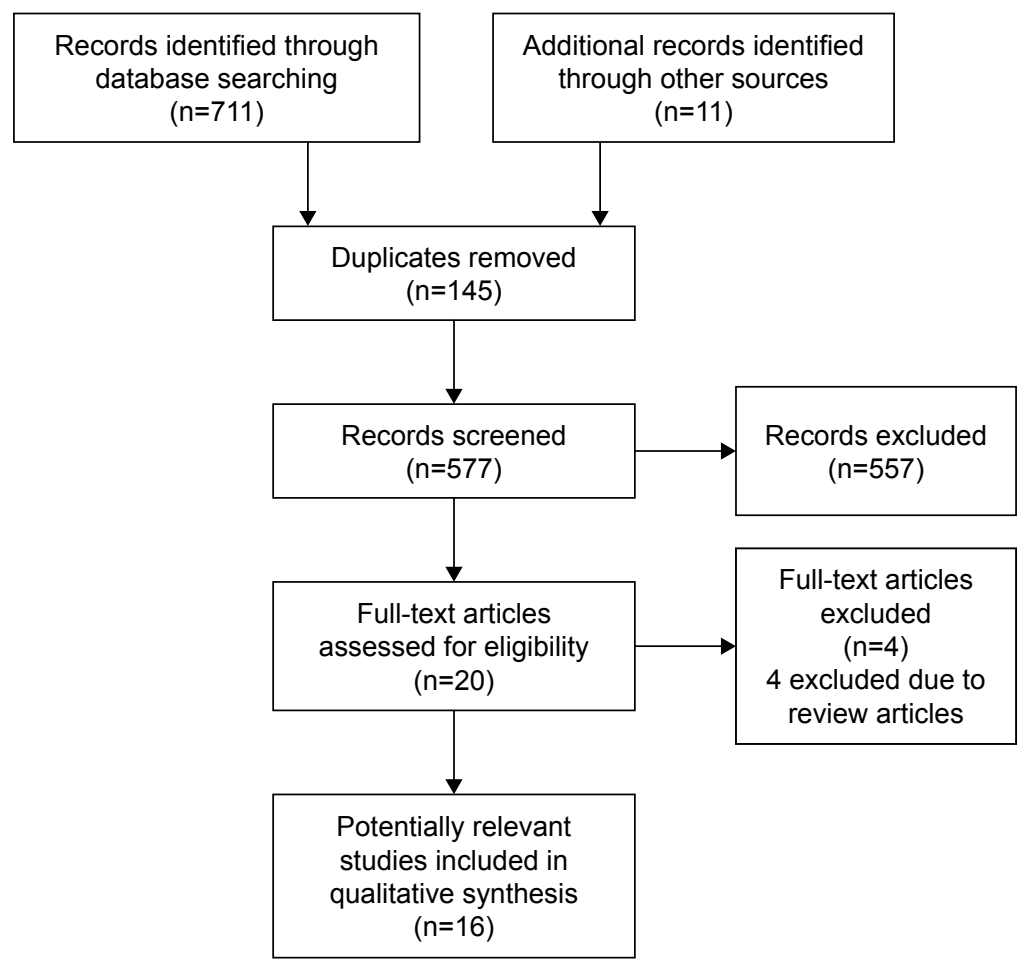

Figure I Prisma flowchart for the selection of studies. 
chikungunya epidemics globally has shown dramatic increase in incidence rate of CHIKV over the last decade. The virus has been reported in more than 60 countries from various WHO regions with approximately one million cases and fatality rate of $0.1 \%$ per year globally. ${ }^{12-14}$

\section{Clinical presentation}

The pathologic mechanism of this virus leads to acute infection of several weeks, which can persist for months with incubation period of 2-12 days. ${ }^{15} \mathrm{CHIKV}$ affects all age groups with dengue-like symptoms characterized by nausea, fever, chills/rigors, headache, polyarthralagia, myalgia, photophobia, and skin rash. ${ }^{8,16}$ The hallmark characteristics of CHIKV infection are saddle-back fever, polyarthralgia (mainly in small joints - ankles, toes, wrists, and phalanges), and a petechial or maculopapular rash (mainly on face, limbs, and trunk). ${ }^{17,18}$ Strikingly, recovery from an acute arthritic illness (high viral load $\sim 10^{8}-10^{9}$ viral copies $/ \mathrm{mL}$ ) takes few days to couple of weeks, though convalescence can be prolonged. In some chronic cases, the clinical manifestations may persist for several months or even years disquieting quality of life. The chronic symptoms mainly include recurrent low-grade body ache, fatigue, neuritis, and persistent swelling and incapacitating inflammation of the joints. ${ }^{19}$ The major factors for CHIKV persistence may include genetic susceptibility, reinfection, exaggerated proinflammatory response leading to tissue damage, evasion of host defense machinery, activation of cryptic virus molecules, and autoimmune reactivity with viral and host antigens. Serious complications are uncommon. However, in rare cases CHIKV can be fatal, primarily in the elderly ( $>65$ years), pregnant women, neonates exposed intrapartum (vertical transmission), young children (especially under 1 year), and persons with preexisting medical conditions like hypertension, diabetes, and heart disorders. Children atypically suffer from severe manifestations of CHIKV disease with neurologic complications like seizures, impaired consciousness, blindness due to retro-bulbar neuritis, and acute flaccid paralysis. ${ }^{20}$

\section{Immunologic basis of CHIKV vaccine development}

CHIKV infection confers life-long immunity. ${ }^{21}$ The activation of innate immune system, in particular, Type I interferons, is fundamental to clearance of acute CHIKV infections. Besides, numerous other innate immune cells like macrophages, natural killer cells, and dendritic cells also play pivotal role as robust responders to early CHIKV infections. However, recently, the protective and pathologic attributes of the innate immunity have been implicated in the pathogenesis of CHIKV infections. ${ }^{22}$ The components of adaptive immunity - both humoral and cellular immunity also imply a crucial role in the protection and elimination of CHIKV-induced infections. However, the humoral immune response (B cell response) plays decisive role in protection against CHIKV infection compared to cellular immunity in human and mouse models. ${ }^{23}$ There are neutralizing antibodies produced through humoral immune mechanism against glycoproteins on the outer surfaces of CHIKV envelope that mediate the clearing of viremia. ${ }^{24}$ Generally, $\operatorname{IgM}$ is detectable between days 2 and 8 of illness and is normally present for only up to 3 months, while convalescent $\mathrm{IgG}$ is measurable from day 4 and persists for years. Recently, Chua et al described that anti-CHIKV IgM targets E1-E2 surface glycoproteins simultaneously rather than E1 or E2 individually, and thus helps in designing more sensitive and reliable IgM assay using an optimized native antigen. ${ }^{25}$ Additionally, $\mathrm{CD}^{+} \mathrm{T}$ cells in cell-mediated immunity play an important role in development of robust protective immunity in controlling acute infections (early phase), whereas $\mathrm{CD} 4^{+} \mathrm{T}$ cells mediate immune response during chronic phase. ${ }^{26}$ However, there is an urgent need for future research to understand the mechanism of immunopathogenesis of adaptive immunity during the sequel of chronic CHIKV infection in humans and vaccine development.

\section{Recent developments in CHIKV vaccines}

The inadequate traditional mosquito control measures, dearth of herd immunity, rapid globalization, sporadic and episodic outbreaks worldwide, and high disease-associated morbidity emphasize the need for research and development of effective vaccines for this neglected tropical disease - "Chikungunya". Vaccines are one of the most important medical contributions and cost-effective public health interventions of the 20th century to prevent communicable diseases. Unlike dengue, the development of an ideal CHIKV vaccine is pragmatic owing to the low antigenic variability of the virus. Despite vigorous efforts and support by researchers, vaccine producers, policy makers, and funding agencies across the globe, no vaccine has been licensed to combat CHIKV infections hitherto. Advances in the field of vaccinology in 21 st century have led to the development of $>20 \mathrm{CHIKV}$ vaccine candidates using diverse vaccine strategies. These include live-attenuated vaccines, whole-virus inactivated vaccines, recombinant virusvectored vaccines, inactivated virus-like particles (VLPs), DNA vaccines, mRNA-based vaccines, synthetic vaccines, and subunit formulations of CHIKV. ${ }^{27-29}$ These CHIKV 
vaccine candidates exhibit wide array of advantages as well as disadvantages in manufacturing, dosage, safety, efficacy, scalable production, and cost effectiveness during preclinical and clinical phases of development. Recently, chikungunya has also been added in the revised list of WHO Research and Development Blueprint preparedness for priority diseases/ pathogens most likely to cause epidemics in the annual review meeting held at Geneva, Switzerland, in January 2017. ${ }^{30}$

In this review, the major breakthroughs in the development of CHIKV vaccines that have entered human clinical trial testing have been discussed in detail. Figure 2 illustrates the various strategies projected for CHIKV vaccine development in humans.

\section{Live-attenuated CHIKV vaccines}

The early attempts to develop CHIKV vaccine began in 1962 with the isolation of a CHIKV strain 15,561 from a viremic patient in Thailand. The wild-type strain was serially passaged by investigators in African green monkey kidney cells at the Walter Reed Army Institute of Research (WRAIR) and in human MRC-5 cells by the US Army Medical Research Institute of Infectious Diseases (USAMRIID) and attenuated strain CHIK 181/Clone 25 was selected as the "vaccine seed". Chikungunya vaccine was manufactured at The Salk Institute-Government Services Division (TSI-GSD) in 1984 in collaboration with the US Department of Defense (DoD). ${ }^{31,32}$ The attenuated TSI-GSD-218 revealed promising results in Phase I and Phase II trials conducted at USAMRIID and the University of Maryland Center for Vaccine Development, respectively. ${ }^{33,34}$ No significant difference in local and systemic reactogenicity parameters between alphavirus-naive volunteers $(n=15)$ and placebo groups was observed in Phase I study. In Phase II randomized, double-blind trial, 73 volunteers (age 18-40 years) were recruited, of which 59 volunteers were immunized with single subcutaneous dose of vaccine and 14 placebos received MRC-5 tissue culture fluid. Forty out of 58 serologically evaluable volunteers (69\%) were seroconverted within 14 days, 57 (98\%) were seroconverted ( $\geq 1: 20$ by PRNT50) by 28 days, and $85 \%$ at 1 year. Five of $59(8 \%)$ vaccinees developed transient mild arthralgia and none was reported by the placebo group $(n=14)$. However, the number and severity of local and systemic reactions were similar in both the groups. Despite safe and highly immunogenic nature in clinical trials, TSI-GSD-218 development was terminated long before origination of deadly outbreaks in 2005 due to lack of funds, uncertainty about safety of bulk production, ambiguity about its misuse as a biologic warfare threat, and limited market for vaccine supply. However, the resurgence of chikungunya in islands of Caribbean and Indian Ocean raised the urgent need of technology transfers of this potential US military CHIKV vaccine. Five companies across the globe signed the material transfer agreement, of which only one pharmaceutical company has successfully developed the vaccine, which shows promising results in preclinical studies, and is ready for Phase I clinical trial. ${ }^{32}$ Strikingly, a recent study by Gorchakov et al has raised the concern of safety issues on the use of attenuated 181/clone $25 \mathrm{CHIV}$ vaccine strain. ${ }^{35}$ Attenuation of strain $181 /$ clone 25 is attained by only two individual point mutations in structural E2 gene and reversions to wild-type sequences may result in phenotypic instability, accounting for mild reactogenic symptoms (transient arthralgia in $8 \%$ of

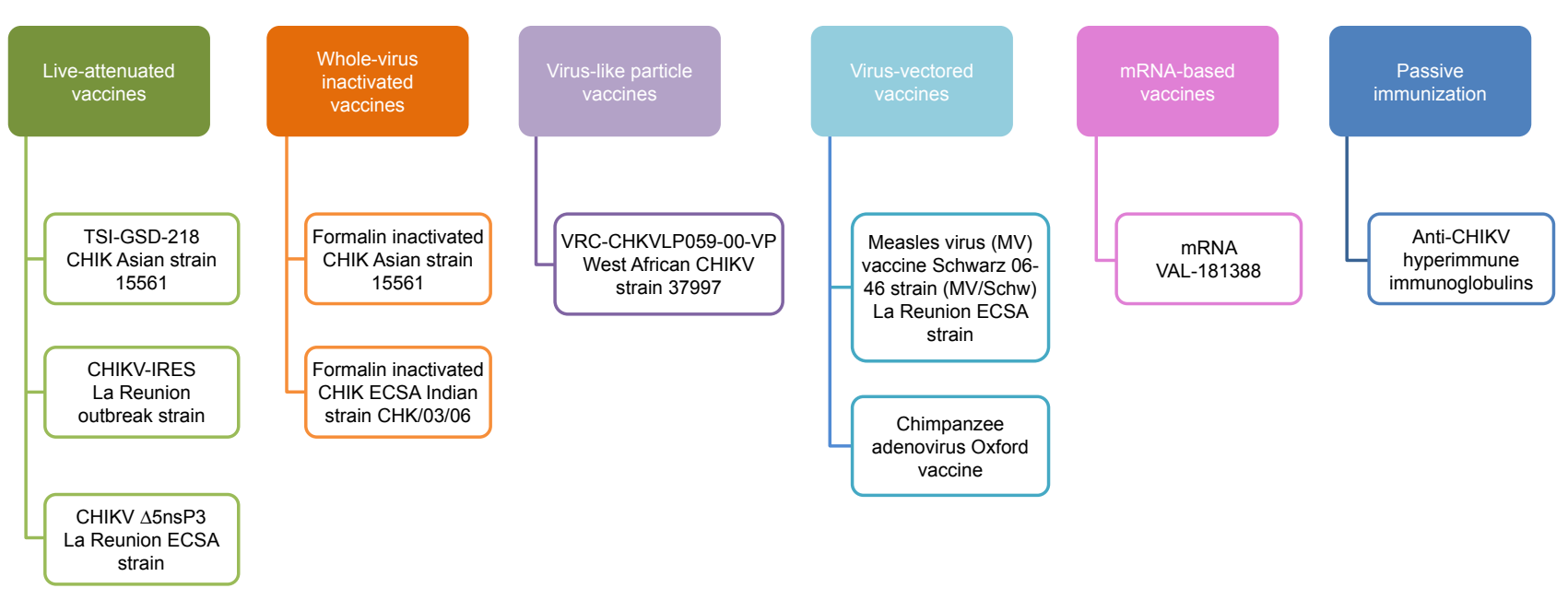

Figure 2 Various strategies projected for chikungunya vaccine development in humans.

Abbreviations: CHIKV-IRES, chikungunya virus-internal ribosomal entry site; ECSA, East/Central/South African; TSI-GSD, The Salk Institute-Government Services Division. 
alphavirus-naïve volunteers), thus emphasizing the need for improvement of 181/clone 25 CHIKV vaccine.

Another novel live-attenuated CHIK/internal ribosomal entry site (IRES) vaccine candidate derived from La Reunion outbreak strain has been developed based on engineering two IRES elements in the CHIKV genome in order to overcome reversion issues of previous vaccine. This vaccine has shown very promising safety and efficacy outcomes in multiple preclinical models. ${ }^{36-39}$ Takeda has recently decided to project this potential CHIK/IRES vaccine candidate toward clinical evaluation. ${ }^{40}$ Over the past year, Zydus Cadila, an Indian health care company, collaborated with the Japanese, Takeda Pharmaceutical Company Limited for conducting joint research to accelerate the development of CHIKV vaccine. The joint venture has raised the hope of rapid advancement of CHIKV/IRES (v1/v2) to human trials in the near future.

Another attenuated vaccine strategy developed to limit reversions is the deletion in structural and nonstructural genes of La Reunion (LR-CHIKV) strain of ECSA genotype. Three different $\mathrm{CHIKV}$ vaccine candidates were developed; $\Delta 5 \mathrm{nsP} 3$ an attenuated virus with a large deletion of 180 nucleotides (60 amino acids) from the $\mathrm{nsP} 3$ replicase region; DREP-E DNAlaunched replicon vaccine with deletion of the region encoding the capsid protein; MVA-CE recombinant vaccinia Ankara virus expressing entire structural (C-E3-E2-6K-E1) protein. A recent study showed that the monovalent, single dose of attenuated $\Delta 5$ virus vaccine was the safest, most efficacious, durable, and generated protective antibody and $\mathrm{T}$ cellular responses with long-term memory in preclinical models. ${ }^{41,42}$ These promising results of attenuated $\Delta 5 \mathrm{nsP} 3$ vaccine candidate named VLA1553 have recently marked its entry into a Phase I trial sponsored by a European biotech company - Valneva, Austria GmbH. ${ }^{43}$

\section{Whole-virus-inactivated $\mathrm{CHIKV}$ vaccines}

The early work on the production of inactivated CHIKV vaccine began in 1967. The whole virus "seed culture" of CHIKV strain 15,561 grown in frozen African green monkey kidney tissue culture cells was inactivated using formalin, followed by purification. ${ }^{44}$ Harrison et al administered formalininactivated vaccine in two doses given twice (either $0.5 \mathrm{~mL}$ or $1 \mathrm{~mL}$ ) in 16 healthy volunteers (aged 21-55 years) divided equally into 2 groups $(n=8) .{ }^{45}$ The results showed $100 \%$ seroconversion rate (plaque reduction neutralization test [PRNT], complement fixation and hemagglutination assays) in both the groups without eliciting any local and systemic reactions. Despite excellent immunogenic response, the development of formalin-killed CHIKV vaccine was halted due to high manufacturing costs involving high biocontainment facilities.
The major drawback was difficulty in comparison of results with other vaccine studies due to difference in dosage of vaccine inoculum used.

After more than three decades, another novel purified, noninfectious, inactivated CHIKV vaccine candidate has been developed using ECSA clinical virus isolates (CHK/03/06) obtained during 2006 outbreak in India and propagated in Vero and MRC-5 cells. The chemical inactivation of stabilized virus formulation was done using formalin or beta-propiolactone. ${ }^{8,46}$ Bharat Biotech International Limited filed several patents (US 8865184, EP 2720715 B1, WO 2017123932 A1) related to inactivated CHIKV vaccine formulation. Subsequently, after successful production and preclinical animal testing, the approved vaccine has advanced for Phase I clinical trial (CTRI/2017/02/007755). ${ }^{47}$

\section{VLP CHIKV vaccines}

In contrast to traditional attenuated and inactivated vaccine approaches, use of VLP has proven to be a new highly efficacious vaccine approach as these are nonreplicating, noninfectious, and self-assembled multiprotein viral constructs resembling native alphavirus. The novel strategy involves transfection of embryonic kidney (HEK-293) cells with optimized codons encoding structural protein genes from West African CHIKV strain 37997, resulting in the production of VLPs with selective expression of E1 and E2 glycoproteins in cell culture supernatant. In preclinical animal model, immunization with these VLPs induces high-titer neutralizing antibodies against enveloped glycoproteins that provide protection against viremia after high-dose challenge with wild-type virus. ${ }^{48,49}$ The novel VLPs vaccine platform became the first recombinant vaccine prototype to enter the Phase I clinical trial (NCT01489358) in 2011. VRC 311 was a nonrandomized, open-label trial in 25 adult volunteers (aged 18-50 years) enrolled at the National Institutes of Health, USA. Participants receive three intramuscular injections of VLP vaccine (VRC-CHKVLP059-00-VP) without adjuvant in three dosage groups 10,20 , or $40 \mu \mathrm{g}$ at weeks 0,4 , and $20 .^{50}$ Overall, vaccine was well tolerated, safe, and highly immunogenic with no serious adverse events (arthralgia). Remarkably, neutralizing antibodies were immunogenic after first and second vaccination in all participants with a significant boost observed 4 weeks after the third vaccination. However, the result of neutralization titers of this study cannot be extrapolated with previous clinical studies as these were measured by high-throughput flow cytometry-based assay as compared to the traditional PRNT assay. Based on the promising results, VLP vaccine has been advanced to 
Phase-IIa randomized multicenter trial involving six cities in endemic regions of the Caribbean (NCT02562482). ${ }^{51}$ The study is sponsored by the National Institutes of Health and designed to recruit 400 healthy volunteers (aged 18-60 years) to evaluate the safety and immunogenicity of $20 \mathrm{mcg}$ dose in a two-injection regimen at days 0 and 28. The primary endpoint results are anticipated to get published soon. Over the past year, PaxVax, a "US based vaccine manufacturing company," has been working to develop a commercial manufacturing process of this potential CHIKV vaccine in alliance with the National Institute of Health and the DoD, USA. PaxVax has in-licensed the chikungunya VLP vaccine platform (PXVX0317) that has entered Phase IIb randomized clinical trial in 415 healthy adults to mitigate the high cost and associated risks (NCT03483961). ${ }^{52}$ Hence, the encouraging results from preclinical and Phase I trial represents VLPs as nonadjuvanated, safe, and highly immunogenic vaccine delivery platform capable of inducing humoral immune response. However, the demerit of this approach is that VLPs require multiple dosages to induce maximal immunogenicity, thereby increasing the cost of manufacturing and limiting the use in resource-constrained countries. Another drawback is their low-yield production. To overcome this issue, Metz and Pijlman in 2016 produced CHIKV-enveloped VLPs in insect cells using recombinant baculovirus expression platform, generating high yields on large scale with high immunogenicity and protection against CHIKV infection in experimental model systems. ${ }^{53}$

\section{Live-attenuated, recombinant virus- vectored $C H I K V$ vaccines}

Another modern vaccine approach uses attenuated measles virus (MV) Schwarz strain (MV/Schw), an RNA virus containing a large $3.7 \mathrm{~kb}$ insert of structural coding gene (C-E3E2-6KE1) derived from La Reunion ECSA CHIKV strain. This recombinant MV-CHIKV vaccine generates VLPs under culture conditions. During preclinical testing, MV-CHIKV induces $100 \%$ seroprotection in mice with strong neutralizing and cellular immune response after second dose. ${ }^{54}$ This novel vaccine is developed by Themis Bioscience $\mathrm{GmbH}$ based on company's proprietary Themaxyn ${ }^{\circledR}$ platform in collaboration with the Institut Pasteur. Based on the promising preclinical data (in mice and unpublished work in nonhuman primates), a Phase I, randomized clinical trial in 42 healthy volunteers (18-45 years) was done to assess the safety, tolerability, and immunogenicity of the recombinant vaccine (EudraCT no 2013-001084-23). ${ }^{55}$ The vaccine was given in three dose groups - low, medium, and high in two immunization schedules. In all dose groups, the candidate vaccine generated adequate neutralizing antibodies after first vaccination and $100 \%$ seroconversion rate following the booster vaccination. The mild-to-moderate adverse events were noticed in all vaccinees; however, no vaccination-related serious adverse events were observed. After the successful Phase I trial, MV-CHIKV is being tested in three Phase II trials conducted simultaneously at three different locations (Europe, USA, and Caribbean island). Recently, the development of MV-CHIKV vaccine is being funded by Innovate UK (a nondepartmental public body, sponsored by the UK Department for Business, Innovation \& Skills) for rapid clinical evaluation at large scale. Themis Bioscience has started a Phase II trial in 2016 to determine the optimum dose of MV-CHIKV vaccine in 320 participants (18-55 years) (NCT02861586). ${ }^{56}$ The study results are anticipated in 2018. Another Phase II trial has also been prompted by Themis Bioscience in collaboration with WRAIR, USA in a previously endemic area in Caribbean island (NCT03101111). ${ }^{57}$ A third Phase II trial of MV-CHIKV vaccine has been actively initiated by NIAID (NCT03028441) in three different states of USA (Georgia, Iowa, and Texas). ${ }^{58}$ Overall, MV-CHIKV vaccine will be tested in more than 600 healthy volunteers. Recently, another Phase II blockrandomized, prospective trial has commenced to compare the different formulations and dosages of MV-CHIKV vaccine in 60 healthy volunteers (NCT). ${ }^{59}$ These active ongoing trials will facilitate the novel MV-CHIKV vaccine candidate toward Phase III trials. The major advantage of this vaccine is its cost-effectiveness in manufacturing on large scale and requires comparably low effective doses in two-dose immunization schedule. However, interference of the preexisting immunity against MV infection or vaccination must be taken into account in upcoming trials.

A novel CHIKV vaccine using advanced recombinant adenoviral technology, known as chimpanzee adenovirus Oxford 1 and 2 (ChAdOx1 and ChAdOx2), expressing structural proteins of CHIKV has been designed by Jenner Institute. Chimpanzee adenoviruses are nonenveloped, genetically stable, complex replication-deficient vectors, thermostable for storage at $45^{\circ} \mathrm{C}$ for 6 months, or ambient temperatures for much longer, and relevant among all age groups. The single dose of this vaccine has elicited safe and robust immune response against the encoded antigens in preclinical models and successfully advanced to good manufacturing practice production. ${ }^{60}$ The research group headed by Prof Arturo Reyes-Sandoval at Jenner Institute, University of Oxford financially supported by Innovate UK has recently projected ChAdOx 1 vaccine to enter nonrandomized Phase I 
clinical trial to evaluate its safety and immunogenicity. ${ }^{61,62}$ This vaccine platform has been recognized by the WHO R\&D Blueprint process for acceleration of large-scale production of CHIKV vaccine in response to outbreak preparedness.

\section{mRNA-based CHIKV vaccines}

Moderna Therapeutics - a US-based Biotech Company (originally named ModeRNA), develops drugs and vaccines based on mRNA technology. The innovative idea behind this promising drug approach is to deliver engineered mRNAs that direct cells to produce and express viral antigenic proteins, emulating a viral infection that could elicit the immune response to generate antibodies. This multidisciplinary mRNA platform overcomes an array of challenges - evasion of immune system against engineered mRNAs, ample synthesis of desired protein inside target cells, and so on. An array of potent vaccines has been developed against emerging infectious diseases like influenza, chikungunya, ebola, and zika using this approach. Single dose of CHIKV mRNA vaccine encoding structural polyprotein of CHIKV provoked $100 \%$ protection in mice and strong antibody responses in nonhuman primates. Based on these promising results, CHIKV vaccine mRNA-1388 has advanced for Phase I study in humans (NCT03325075). ${ }^{63}$

\section{Passive immunization}

The passive immunization by transfer of purified polyclonal immunoglobulins from human sera has been widely demonstrated in many viral infections. Couderc et al used human immunoglobulins purified from convalescent phase plasma samples of CHIKV-infected patients in two susceptible mouse models. ${ }^{64}$ These immunoglobulins show high neutralizing activity in vitro, prevent viral dissemination in tissues, and show prophylactic and therapeutic efficacy from lethal CHIKV infections. However, over the past years, a number of studies have also established that monoclonal antibodies also show protective efficacy in CHIKV-infected mice. ${ }^{65-68}$ These studies suggested that CHIKV immunoglobulins may act as antiviral therapy for high-risk human population exposed to CHIKV. ${ }^{69}$ Keeping these studies in view, anti-CHIKV hyperimmune intravenous immunoglobulins therapy undergoes an open, nonrandomized Phase I/II trial (CHIKIVIG-01) in high-risk neonates born to CHIKV-viremic mothers (NCT02230163). ${ }^{70}$

\section{Vaccine trials in clinical pipeline}

Work over the past 5 years has brought revolution in the CHIKV vaccine development; predominantly 2017/2018 has marked the crucial year of this decade in which eleven promising clinical trials have been instigated in different phases of clinical pipeline worldwide. Table 1 summarizes the various chikungunya vaccine strategies in human clinical trials.

\section{Ongoing Phase I clinical trials}

1. A novel CHIKV live-attenuated vaccine VLA1553 $(\Delta 5 \mathrm{~ns} 3)$ has been projected for Phase I, randomized, multicenter trial in 120 healthy volunteers of age $18-45$ years to be enrolled across three locations in USA (ClinicalTrials.gov NCT03382964). Single immunization of three dose levels - low $(n=30)$, medium $(n=30)$, and high $(n=60)$ will be given intramuscularly to assess the safety and immunogenicity. This study has been sponsored by Valneva Austria GmbH. ${ }^{43}$

2. Another Phase I, randomized, open-label, placebocontrolled, multicentric clinical trial has started recruiting healthy adults $(n=60)$ of age $18-50$ years to evaluate the safety, tolerability, and immunogenicity of inactivated CHIKV vaccine BBV87 in prime-boost regimen. This trial has been registered by Bharat Biotech International Ltd, Hyderabad with Clinical Trial Registry India (CTRI/2017/02/007755) in early 2017. ${ }^{47}$

3. A Phase I/II, double blinded, placebo-controlled, dose comparison trial (ClinicalTrials.gov NCT03028441) has started recruiting healthy adults aged $18-45$ years $(n=180)$ to evaluate the safety, immunogenicity, and schedule of live-attenuated, measles-vectored CHIKV vaccine (MV-CHIKV) developed by Themis Bioscience $\mathrm{GmbH}$. The different dosage and immunization schedules will be evaluated. Themis' prophylactic vaccine candidate (MV-CHIKV) is being tested and sponsored by NIAID, USA. ${ }^{58}$

4. The Jenner Institute, University of Oxford has designed a novel chimpanzee adenoviral vector-based vaccine, ChAdOx1- and ChAdOx2-expressing chikungunya structural antigens (capsid and envelope glycoprotein). After the preclinical validation of the single-dose, adjuvant-free CHIKV vaccine, this novel candidate vaccine has now entered into a Phase I clinical trial funded by UK's Innovate agency ${ }^{61}$ An open-label, nonrandomized, dose-escalation trial has started recruiting healthy volunteers (age 18-50 years) to assess the safety and immunogenicity of ChAdOx1 CHIK vaccine (CHIK001) (ClinicalTrials.gov NCT03590392). ${ }^{62}$

5. In late 2017, a Phase I, randomized, placebo-controlled, dose-escalation clinical trial (ClinicalTrials.gov 
Table I Chikungunya vaccine strategies in human clinical trial testing

\begin{tabular}{|c|c|c|c|c|c|}
\hline Type of vaccine & Manufacturer & $\begin{array}{l}\text { Phase of } \\
\text { clinical trial }\end{array}$ & Trial registration no & \begin{tabular}{|l} 
Recruitment \\
status
\end{tabular} & Reference \\
\hline \multicolumn{6}{|l|}{ Live-attenuated vaccine } \\
\hline \multirow[t]{2}{*}{ TSI-GSD-2। 8} & \multirow[t]{2}{*}{$\begin{array}{l}\text { USAMRIID/Salk Institute for } \\
\text { Biological Studies }\end{array}$} & Phase I & NA & $\begin{array}{l}\text { Completed } \\
\text { with results }\end{array}$ & 33 \\
\hline & & Phase II & NA & $\begin{array}{l}\text { Completed } \\
\text { with results }\end{array}$ & 34 \\
\hline$\Delta 5$ nsP3 VLAI 553 & Valneva Austria GmbH & Phase I & ClinicalTrials.gov NCT03382964 & Recruiting & 43 \\
\hline \multicolumn{6}{|l|}{ Inactivated vaccine } \\
\hline $\begin{array}{l}\text { Formalin-inactivated } \\
\text { vaccine }\end{array}$ & $\begin{array}{l}\text { United States USAMRIID and } \\
\text { WRAIR, USA }\end{array}$ & Phase I & NA & $\begin{array}{l}\text { Completed } \\
\text { with results }\end{array}$ & 45 \\
\hline $\begin{array}{l}\text { Inactivated chikungunya } \\
\text { vaccine BBV87 }\end{array}$ & $\begin{array}{l}\text { Bharat Biotech International, } \\
\text { Ltd, India }\end{array}$ & Phase I & $\begin{array}{l}\text { Clinical Trial Registry India } \\
\text { (CTRI/20I7/02/007755) }\end{array}$ & $\begin{array}{l}\text { Active, } \\
\text { recruiting }\end{array}$ & 47 \\
\hline \multicolumn{6}{|l|}{ VLP vaccine } \\
\hline $\begin{array}{l}\text { VRC3 I I: VRC- } \\
\text { CHKVLP059-00-VP }\end{array}$ & NIAID & Phase I & ClinicalTrials.gov NCT0I489358 & $\begin{array}{l}\text { Completed } \\
\text { with results }\end{array}$ & 50 \\
\hline $\begin{array}{l}\text { VRC704: VRC- } \\
\text { CHKVLP059-00-VP }\end{array}$ & NIAID & Phase lla & ClinicalTrials.gov NCT02562482 & $\begin{array}{l}\text { Active, not } \\
\text { recruiting }\end{array}$ & 51 \\
\hline PXVX03।7 CHIKV-VLP & Pax Vax & Phase Ilb & ClinicalTrials.gov NCT0348396I & $\begin{array}{l}\text { Active, not } \\
\text { recruiting }\end{array}$ & 52 \\
\hline \multicolumn{6}{|c|}{ Live, measles-virus-vectored vaccine } \\
\hline MV-CHIKV & $\begin{array}{l}\text { Themis Bioscience GmbH/ } \\
\text { Institut Pasteur in Paris, in } \\
\text { collaboration with University } \\
\text { of Vienna, Austria }\end{array}$ & Phase I & EudraCT 20I3-00I084-23 & $\begin{array}{l}\text { Completed } \\
\text { with results }\end{array}$ & 55 \\
\hline \multirow[t]{2}{*}{ MV-CHIK-202 } & \multirow{2}{*}{$\begin{array}{l}\text { Themis Bioscience } \mathrm{GmbH} \text {, in } \\
\text { collaboration with University } \\
\text { of Vienna, Austria }\end{array}$} & \multirow[t]{2}{*}{ Phase II } & Edura CT 2015-004037-26 & Completed & \multirow[t]{2}{*}{56} \\
\hline & & & ClinicalTrials.gov NCT0286I586 & Results awaited & \\
\hline MV-CHIK-204 & $\begin{array}{l}\text { Themis Bioscience GmbH in } \\
\text { collaboration with WRAIR, } \\
\text { USA }\end{array}$ & Phase II & ClinicalTrials.gov NCT03I0IIII & $\begin{array}{l}\text { Active, not } \\
\text { recruiting }\end{array}$ & 57 \\
\hline MV-CHIKV & $\begin{array}{l}\text { Themis Bioscience } \mathrm{GmbH} \text { in } \\
\text { collaboration with NIAID }\end{array}$ & Phase I/II & ClinicalTrials.gov NCT0302844I & $\begin{array}{l}\text { Active, not } \\
\text { recruiting }\end{array}$ & 58 \\
\hline MV-CHIKV & $\begin{array}{l}\text { Themis Bioscience in } \\
\text { collaboration with Celerion } \\
\text { Belfast }\end{array}$ & Phase II & ClinicalTrials.gov NCT03635086 & $\begin{array}{l}\text { Active, not } \\
\text { recruiting }\end{array}$ & 59 \\
\hline $\begin{array}{l}\text { Chimpanzee adenovirus- } \\
\text { vectored vaccine }\end{array}$ & $\begin{array}{l}\text { University of Oxford UK } \\
\text { Research and Innovation }\end{array}$ & Phase I & $\begin{array}{l}\text { UK Research and Innovation } \\
972212\end{array}$ & Active & 62 \\
\hline \multicolumn{6}{|l|}{ mRNA-based vaccine } \\
\hline mRNA VAL- 181388 & $\begin{array}{l}\text { Moderna Therapeutics, } \\
\text { USA and Defense Advanced } \\
\text { Research Projects Agency }\end{array}$ & Phase I & ClinicalTrials.gov NCT03325075 & Recruiting & 63 \\
\hline \multicolumn{6}{|l|}{ Passive immunization } \\
\hline $\begin{array}{l}\text { Anti-CHIKV } \\
\text { hyperimmune } \\
\text { immunoglobulins }\end{array}$ & $\begin{array}{l}\text { Centre Hospitalier } \\
\text { Universitaire de, Pointe-à- } \\
\text { Pitre, Caribbean }\end{array}$ & Phase I/II & ClinicalTrials.gov NCT02230I63 & Unknown & 70 \\
\hline
\end{tabular}

Abbreviations: MV, measles virus; NA, not applicable; NIAID, National Institute of Allergy and Infectious Diseases; USAMRIID, United States Army Medical Research Institute of Infectious Diseases; TSI-GSD, The Salk Institute-Government Services Division; VLP, virus-like particle; WRAIR, Walter Reed Army Institute of Research.

NCT03325075) to evaluate the safety and immunogenicity of CHIKV vaccine candidate dubbed mRNA VAL-181388 in healthy adults $(n=60)$ in a nonendemic chikungunya region has been registered. The study involves three dosages in two immunization schedules. This study is sponsored by Moderna therapeutics supported by Defense Advanced Research Projects Agency, an agency of the US DoD. ${ }^{63}$ 
6. An open, nonrandomized Phase I/II trial (ClinicalTrials. gov NCT02230163) has been initiated in 2014, with an aim to evaluate the safety and clinical evaluation of anti-CHIKV hyperimmune intravenous immunoglobulins (CHIKIVIG-01) therapy to high-risk neonates born to CHIKV-viremic mothers. Unfortunately, very few neonates have been recruited till date and study has not been verified since last 2 years. $^{70}$

7. Another commercial organization, Indian Immunologicals Ltd, Hyderabad has received chikungunya vaccine seed materials (TSI-GSD-218 or 181/clone 25) from the USAMRIID. After the completion of initial preclinical toxicity studies and reinitiating the inactivated CHIKV vaccine development, the Phase I clinical trial is in progress. $^{71}$

\section{Ongoing Phase II clinical trials}

1. After the promising results of Phase I trial of VLP vaccine that consists of the E1, E2, and capsid proteins published by Chang et al, ${ }^{50}$ a Phase II, multicenter, randomized, placebo-controlled, double-blind trial of CHIKV VLP vaccine has been registered by NIAID (ClinicalTrials.gov NCT02562482) in 400 healthy adults (age 18-60 years) in CHIKV endemic areas. The primary objectives are to evaluate safety and tolerability of a two-injection vaccine regimen of VRC-CHKVLP059-00-VP at $20 \mathrm{mcg}$ compared to placebo (PBS) in healthy adults. ${ }^{51}$

2. Pax Vax has recently announced a Phase II parallelgroup, randomized, double-blind trial (ClinicalTrials.gov NCT03483961) to evaluate the safety and immunogenicity of VLP vaccine, PXVX0317 CHIKV-VLP, to different doses/formulations and schedules in 415 healthy adults (age 18-45 years) at three locations in USA. This trial has received the designation of priority access "Fast Track Program" from Food and Drug Administration of USA. ${ }^{52}$

3. Data from the Phase I trial of MV-based CHIKV vaccine trial are greatly anticipated. Recently, a Phase II, double-blinded, block-randomized, and placebo-controlled trial has been registered (ClinicalTrials.gov NCT02861586) to evaluate the optimal dose of measles-virus vaccine (MV-CHIK-202) by assessing immunogenicity, safety, and tolerability in 320 healthy volunteers (age 18-55 years). Two dose levels (low and high) at three immunization schedules will be assessed. This trial is being sponsored by Themis Bioscience $\mathrm{GmBH}$ in collaboration with University of Vienna, Austria. Very recently, the ongoing trial has completed the recruitment of the participants and 263 healthy adults have been enrolled. The results are expected to be published soon. ${ }^{56}$

4. Another Phase II, comparator-controlled, randomized, double-blinded clinical trial (ClinicalTrials.gov NCT03101111) has started recruiting $(n=100)$ healthy volunteers aged 21-50 years in a previously epidemic area (Puerto Rico) to evaluate the safety of live-attenuated MV-vectored CHIKV vaccine (MV-CHIK204). This trial is also sponsored by Themis Bioscience $\mathrm{GmBH}$ in collaboration with WRAIR. ${ }^{57}$

5. Themis Bioscience has initiated an additional Phase II randomized trial to investigate the safety, tolerability, and long-term durability of licensed MV-CHIKV in different formulations (lyophilized, liquid frozen, and liquid SPS) and dose regimens. ${ }^{59}$

\section{Challenges in CHIKV vaccine development} The attempts to develop CHIKV vaccine began in the early 1950s. The development of a safe and effective vaccine has been plagued by several challenges and quandaries. Unlike global dengue vaccine development for which more than 60 clinical trials have been registered so far, only few CHIKV vaccine development initiatives have been completed, with limited vaccine candidates in preclinical and clinical pipeline using different technologic approaches. The challenges in developing a CHIKV vaccine can be categorized into three broad groups: clinical/therapeutic, social/demographic, and economic.

The major issue in the CHIKV vaccine field is the selection of biologic assays used to evaluate vaccine immunogenicity. The standard protection assay, that is, PRNT, is usually based on the measurement of neutralizing antibodies at baseline and post-vaccination. The interlab and interassay cutoff variation (two to threefold) in seroconversion rates and antibody titers exhibit significant shortcoming in vaccine trials. The low and waning immunogenicity of candidate vaccines in vaccinees and healthy controls is also a matter of great concern. The candidate vaccines in pipeline mainly focus on adult population, so their efficacy in pediatric population remains uncertain. The appropriate dosing requirement of varied $\mathrm{CHIKV}$ vaccine formulations, that is, multiple doses or single dose is better to maintain immunity against CHIKV, is also vacillating. Also, the substantial gap in our understanding of innate and adaptive immune responses that confer protection or pathologic immune response following a CHIKV infection poses another major challenge in vaccine development. The codistribution and coinfection of chikungunya and dengue virus outbreaks is another bottleneck. 
Despite the substantial increase in global travel to many CHIKV-endemic areas, the unpredictable epidemiology of chikungunya (sporadic outbreaks interspersed by long periods of quiescence) makes it less attractive for travel and tourism industry unlike diseases like yellow fever. The prevalence of chikungunya among the developing and underdeveloped countries with low literary levels, low per capita income, low living standards, and poor sanitation reduces the probability of marketing and commercialization of CHIKV vaccine in developed nations. Finally, a successful CHIKV vaccine candidate to enter the Phase III clinical trials and licensure is challenging due to selection of a suitable location with adequate incidence of chikungunya in target population to demonstrate clinical efficacy. The huge capital investment, uncertain profits, and redundant regulatory pathways further reduce the interest of pharmaceutical companies and investors in CHIKV vaccine development. However, to overcome the financial risks and regulatory challenges, there is an urgent need for the global partnerships including national and international public, private, and government funding agencies, industry, researchers, clinicians, public health officials, and other global health stakeholders with an aim to develop new vaccines.

\section{Summary}

This review gives an insight about several CHIKV vaccines in clinical pipeline and puts into perspective the findings, merits, and limitations of all clinical trials currently available. Although no vaccine is licensed at present, an effective vaccine for chikungunya will not only deter the rising trend of this infection but also contribute in limiting the disease-associated morbidity and health care costs involved in its management. In general, the ideal CHIKV vaccine to be licensed and ready for commercial use should be well tolerated, safe, adjuvant-free, and cost-effective, devoid of major vaccine-related adverse events such as arthralgia, have robust efficacy, and provide long-lasting immunity after a single dose in the general population. The efficacy of these vaccines in an endemic setting with limited resources would be greatly valuable as a significant burden of chikungunya lies in such regions.

This study will be helpful in suggesting which type of vaccine is clinically relevant and could be adopted for future clinical trials for estimation of safety, immunogenicity, tolerability, and efficacy. The rapid ongoing progress has raised hope for large-scale Phase III efficacy trials in endemic settings to begin soon. Although there is still a long way to go before a prophylactic vaccine for chikungunya is available, the vigorous restorative advancement during the last year makes the likelihood of having an effective vaccine in the near future high.

\section{Acknowledgments}

This work was supported by the Department of Health Research, New Delhi, and the Advanced Centre for Evidence Based Child Health, Postgraduate Institute of Medical Education and Research, Chandigarh, set up by Indian Council of Medical Research, New Delhi, India.

\section{Disclosure}

Vishavdeep Goyal is the Chief Operating Officer of Apollo Hospitals International Limited, Ahmedabad, India. The authors report no other conflicts of interest in this work.

\section{References}

1. Epstein PR. Chikungunya fever resurgence and global warming. Am J Trop Med Hyg. 2007;76(3):403-404.

2. Weaver SC, Lecuit M. Chikungunya virus and the global spread of a mosquito-borne disease. N Engl J Med. 2015;372(13):1231-1239.

3. Powers AM, Brault AC, Tesh RB, Weaver SC. Re-emergence of chikungunya and O'nyong-nyong viruses: evidence for distinct geographical lineages and distant evolutionary relationships. J Gen Virol. 2000;81(Pt 2):471-479.

4. Diallo M, Thonnon J, Traore-Lamizana M, Fontenille D. Vectors of chikungunya virus in Senegal: current data and transmission cycles. Am J Trop Med Hyg. 1999;60(2):281-286.

5. Tsetsarkin KA, Vanlandingham DL, Mcgee CE, Higgs S. A single mutation in chikungunya virus affects vector specificity and epidemic potential. PLoS Pathog. 2007;3(12):e201.

6. Thiboutot MM, Kannan S, Kawalekar OU, et al. Chikungunya: a potentially emerging epidemic? PLoS Negl Trop Dis. 2010;4(4):e623.

7. Dash PK, Parida MM, Santhosh SR, et al. East Central South African genotype as the causative agent in reemergence of chikungunya outbreak in India. Vector Borne Zoonotic Dis. 2007;7(4):519-528.

8. Pialoux G, Gaüzère BA, Jauréguiberry S, Strobel M. Chikungunya, an epidemic arbovirosis. Lancet Infect Dis. 2007;7(5):319-327.

9. Roth A, Hoy D, Horwood PF, et al. Preparedness for threat of chikungunya in the Pacific. Emerg Infect Dis. 2014;20(8):e130696.

10. Yactayo S, Staples JE, Millot V, Cibrelus L, Ramon-Pardo P. Epidemiology of chikungunya in the Americas. J Infect Dis. 2016; 214(suppl 5):S441-S445.

11. Petersen LR, Powers AM. Chikungunya: epidemiology. F1000Res. 2016;5:F1000 Faculty Rev-82.

12. WHO. Chikungunya; fact sheet [2017]. Available from: http://www.who. int/news-room/fact-sheets/detail/chikungunya. Accessed February 20, 2018 .

13. Josseran L, Paquet C, Zehgnoun A, et al. Chikungunya disease outbreak, Reunion Island. Emerg Infect Dis. 2006;12(12):1994-1995.

14. Morrison TE. Reemergence of chikungunya virus. J Virol. 2014;88(20): 11644-11647.

15. Schmaljohn AL, McClain D. Alphaviruses (Togaviridae) and flaviviruses (Flaviviridae). In: Baron S, editor. Medical Microbiology. 4th ed. Galveston, TX: University of Texas Medical Branch at Galveston; 1996.

16. Schwartz O, Albert ML. Biology and pathogenesis of chikungunya virus. Nat Rev Microbiol. 2010;8(7):491-500.

17. Lakshmi V, Neeraja M, Subbalaxmi MV, et al. Clinical features and molecular diagnosis of chikungunya fever from South India. Clin Infect Dis. 2008;46(9):1436-1442. 
18. Vijayakumar KP, Nair Anish TS, George B, Lawrence T, Muthukkutty SC, Ramachandran R. Clinical profile of chikungunya patients during the epidemic of 2007 in Kerala, India. J Glob Infect Dis. 2011;3(3):221-226.

19. Dupuis-Maguiraga L, Noret M, Brun S, Le Grand R, Gras G, Roques P. Chikungunya disease: infection-associated markers from the acute to the chronic phase of arbovirus-induced arthralgia. PLoS Negl Trop Dis. 2012;6(3):e1446.

20. Sebastian MR, Lodha R, Kabra SK. Chikungunya infection in children. Indian J Pediatr. 2009;76(2):185-189.

21. Tiwari M, Parida M, Santhosh SR, Khan M, Dash PK, Rao PV. Assessment of immunogenic potential of Vero adapted formalin inactivated vaccine derived from novel ECSA genotype of chikungunya virus. Vaccine. 2009;27(18):2513-2522.

22. Long KM, Heise MT, Mark TH. Protective and pathogenic responses to chikungunya virus infection. Curr Trop Med Rep. 2015;2(1):13-21.

23. Lum FM, Teo TH, Lee WW, Kam YW, Rénia L, Ng LF. An essential role of antibodies in the control of chikungunya virus infection. J Immunol. 2013;190(12):6295-6302.

24. Fong RH, Banik SS, Mattia K, et al. Exposure of epitope residues on the outer face of the chikungunya virus envelope trimer determines antibody neutralizing efficacy. $J$ Virol. 2014;88(24):14364-14379.

25. Chua CL, Sam IC, Chiam CW, Chan YF. The neutralizing role of IgM during early chikungunya virus infection. PLoS One. 2017;12(2): e0171989.

26. Wauquier N, Becquart P, Nkoghe D, Padilla C, Ndjoyi-Mbiguino A, Leroy EM. The acute phase of chikungunya virus infection in humans is associated with strong innate immunity and T CD8 cell activation. J Infect Dis. 2011;204(1):115-123.

27. Singh $P$, Chhabra M, Mittal V. Current research and clinical trials for a vaccine against chikungunya virus. Vaccine: Develop Ther. 2013;3:35-46.

28. Schwameis M, Buchtele N, Wadowski PP, Schoergenhofer C, Jilma B. Chikungunya vaccines in development. Hum Vaccin Immunother. 2016; 12(3):716-731.

29. Powers AM. Vaccine and therapeutic options to control chikungunya virus. Clin Microbiol Rev. 2018;31(1):e001104-e01116.

30. WHO Research and Development Blueprint. 2017 Annual review of diseases prioritized under the Research and Development Blueprint [2017]. Available from: http://www.who.int/blueprint/what/researchdevelopment/2017-Prioritization-Long-Report.pdf?ua=1. Accessed April 21, 2018.

31. Levitt NH, Ramsburg HH, Hasty SE, Repik PM, Cole FE, Lupton HW. Development of an attenuated strain of chikungunya virus for use in vaccine production. Vaccine. 1986;4(3):157-162.

32. Hoke CH, Pace-Templeton J, Pittman P, et al. US Military contributions to the global response to pandemic chikungunya. Vaccine. 2012;30(47): 6713-6720.

33. Mcclain DJ, Pittman PR, Ramsburg HH, et al. Immunologic interference from sequential administration of live attenuated alphavirus vaccines. J Infect Dis. 1998;177(3):634-641.

34. Edelman R, Tacket CO, Wasserman SS, Bodison SA, Perry JG, Mangiafico JA. Phase II safety and immunogenicity study of live chikungunya virus vaccine TSI-GSD-218. Am J Trop Med Hyg. 2000; 62(6):681-685.

35. Gorchakov R, Wang E, Leal G, et al. Attenuation of chikungunya virus vaccine strain 181 /clone 25 is determined by two amino acid substitutions in the E2 envelope glycoprotein. $J$ Virol. 2012;86(11): 6084-6096.

36. Plante K, Wang E, Partidos CD, et al. Novel chikungunya vaccine candidate with an IRES-based attenuation and host range alteration mechanism. PLoS Pathog. 2011;7(7):e1002142.

37. Chu H, Das SC, Fuchs JF, et al. Deciphering the protective role of adaptive immunity to CHIKV/IRES a novel candidate vaccine against chikungunya in the A129 mouse model. Vaccine. 2013;31(33):3353-3360.

38. Roy CJ, Adams AP, Wang E, et al. Chikungunya vaccine candidate is highly attenuated and protects nonhuman primates against telemetrically monitored disease following a single dose. J Infect Dis. 2014;209(12): 1891-1899.
39. Seymour RL, Adams AP, Leal G, Alcorn MD, Weaver SC. A rodent model of chikungunya virus infection in RAG1 -/- mice, with features of persistence, for vaccine safety evaluation. PLoS Negl Trop Dis. 2015; 9(6): $\mathrm{e} 0003800$.

40. Ahola T, Couderc T, Courderc T, et al. Therapeutics and vaccines against chikungunya virus. Vector Borne Zoonotic Dis. 2015;15(4):250-257.

41. HallengärdD, Kakoulidou M, Lulla A, et al. Novel attenuated chikungunya vaccine candidates elicit protective immunity in C57BL/6 mice. J Virol. 2014;88(5):2858-2866.

42. Roques P, Ljungberg K, Kümmerer BM, et al. Attenuated and vectored vaccines protect nonhuman primates against chikungunya virus. JCI Insight. 2017;2(6):e83527.

43. ClinicalTrials.gov. A randomized, observer-blinded, dose-escalation Phase 1 study to assess the safety and immunogenicity of three different dose levels of a live-attenuated chikungunya virus vaccine candidate (VLA1553) in healthy volunteers aged 18 to 45 years. Available from: https://clinicaltrials.gov/ct2/show/record/NCT03382964. Accessed March 3, 2018.

44. Harrison VR, Marshall JD, Guilloud NB. The presence of antibody to chikungunya and other serologically related viruses in the sera of sub-human primate imports to the United States. J Immunol. 1967;98(5):979-981.

45. Harrison VR, Eckels KH, Bartelloni PJ, Hampton C. Production and evaluation of a formalin-killed chikungunya vaccine. J Immunol. 1971; 107(3):643-647.

46. Kumar M, Sudeep AB, Arankalle VA. Evaluation of recombinant E2 protein-based and whole-virus inactivated candidate vaccines against chikungunya virus. Vaccine. 2012;30(43):6142-6149.

47. CTRI/2017/02/007755. Phase-I open label, dose-escalation clinical trial to evaluate the safety, tolerability and immunogenicity of chikungunya vaccine in healthy adults of 18 to 50 years age. Available from: http:// ctri.nic.in/Clinicaltrials/pdf_generate.php?trialid $=14549 \&$ EncHid $=$ $\&$ modid $=\&$ compid $=\% 27, \% 2714549 \operatorname{det} \% 27$. Accessed November 5, 2017.

48. Akahata W, Yang ZY, Andersen $\mathrm{H}$, et al. A virus-like particle vaccine for epidemic chikungunya virus protects nonhuman primates against infection. Nat Med. 2010;16(3):334-338.

49. Akahata W, Nabel GJ. A specific domain of the chikungunya virus E2 protein regulates particle formation in human cells: implications for alphavirus vaccine design. $J$ Virol. 2012;86(16):8879-8883.

50. Chang LJ, Dowd KA, Mendoza FH, et al. Safety and tolerability of chikungunya virus-like particle vaccine in healthy adults: a phase 1 dose-escalation trial. Lancet. 2014;384(9959):2046-2052.

51. ClinicalTrials.gov. Phase 2 randomized, placebo-controlled trial to evaluate the safety and immunogenicity of a chikungunya virus-like particle vaccine, VRC-CHKVLP059-00-VP, in healthy adults. Available from: https://clinicaltrials.gov/ct2/show/record/NCT02562482. Accessed October 25, 2017.

52. ClinicalTrials.gov. A Phase 2 parallel-group, randomized, doubleblind study to assess the safety and immunogenicity of PXVX0317 (chikungunya virus Virus-Like Particle vaccine [CHIKV-VLP], unadjuvanted or alum-adjuvanted). Available from: https://clinicaltrials. gov/ct2/show/record/NCT03483961. Accessed June 4, 2018.

53. Metz SW, Pijlman GP. Production of chikungunya Virus-Like Particles and subunit vaccines in insect cells. Methods Mol Bio. 2016;1426:297-309.

54. Brandler S, Ruffié C, Combredet C, et al. A recombinant measles vaccine expressing chikungunya virus-like particles is strongly immunogenic and protects mice from lethal challenge with chikungunya virus. Vaccine. 2013;31(36):3718-3725.

55. Ramsauer K, Schwameis M, Firbas C, et al. Immunogenicity, safety, and tolerability of a recombinant measles-virus-based chikungunya vaccine: a randomised, double-blind, placebo-controlled, active-comparator, first-in-man trial. Lancet Infect Dis. 2015;15(5):519-527.

56. ClinicalTrials.gov. Double-blinded, randomized, priorix ${ }^{\circledR}$ - and placebocontrolled, trial to evaluate the optimal dose of MV-CHIK vaccine (against chikungunya virus) in regard to immunogenicity, safety and tolerability in healthy volunteers. Available from: https://clinicaltrials. gov/ct2/show/record/NCT02861586. Accessed October 23, 2017. 
57. ClinicalTrials.gov. Phase 2 study of a live attenuated measles virusvectored chikungunya vaccine in a previously epidemic area. Available from: https://clinicaltrials.gov/ct2/show/record/NCT03101111. Accessed November 8, 2017.

58. ClinicalTrials.gov. A Phase 1, double-blinded, placebo-controlled, dose comparison trial to evaluate the safety, immunogenicity and schedule of measles-vectored virus chikungunya vaccine (MV-CHIK) in healthy adults. Available from: https://clinicaltrials.gov/ct2/show/ record/NCT03028441. Accessed November 25, 2017.

59. ClinicalTrials.gov. Safety, tolerability and long-term immunogenicity of different formulations of a chikungunya vaccine. Available from: https:/clinicaltrials.gov/ct2/show/study/NCT03635086. Accessed September 12, 2018.

60. Ewer K, Sebastian S, Spencer AJ, Gilbert S, Hill AVS, Lambe T. Chimpanzee adenoviral vectors as vaccines for outbreak pathogens. Hum Vaccin Immunother. 2017;13(12):3020-3032.

61. UK Research and Innovation. A Phase-I clinical trial of a chikungunya vaccine using a single dose and no adjuvant. Available from: https:// gtr.ukri.org/projects?ref=972212. Accessed December 5, 2017.

62. ClinicalTrials.gov. Safety and immunogenicity of a candidate CHIKV vaccine. Available from: https://clinicaltrials.gov/ct2/show/ NCT03590392. Accessed September 10, 2018.

63. ClinicalTrials.gov. A Phase 1, randomized, placebo-controlled, doseranging study to evaluate the safety and immunogenicity of VAL-181388 in healthy adults in a non endemic chikungunya region. Available from: https://clinicaltrials.gov/ct2/show/record/NCT03325075. Accessed January 4, 2017.
64. Couderc T, Khandoudi N, Grandadam M, et al. Prophylaxis and therapy for chikungunya virus infection. J Infect Dis. 2009;200(4): 516-523.

65. Fric J, Bertin-Maghit S, Wang CI, Nardin A, Warter L. Use of human monoclonal antibodies to treat chikungunya virus infection. $J$ Infect Dis. 2013;207(2):319-322.

66. Selvarajah S, Sexton NR, Kahle KM, et al. A neutralizing monoclonal antibody targeting the acid-sensitive region in chikungunya virus E2 protects from disease. PLoS Negl Trop Dis. 2013;7(9):e2423.

67. Pal P, Dowd KA, Brien JD, et al. Development of a highly protective combination monoclonal antibody therapy against chikungunya virus. PLoS Pathog. 2013;9(4):e1003312.

68. Miner JJ, Cook LE, Hong JP, et al. Therapy with CTLA4-Ig and an antiviral monoclonal antibody controls chikungunya virus arthritis. Sci Transl Med. 2017;9(375):e3438.

69. Clayton AM. Monoclonal antibodies as prophylactic and therapeutic agents against chikungunya virus. J Infect Dis. 2016;214(suppl 5): S506-S509.

70. ClinicalTrials.gov. Prevention of chikungunya infection in neonates: clinical evaluation of anti-CHIKV hyperimmune intravenous immunoglobulins. Available from: https://clinicaltrials.gov/ct2/show/record/ NCT02230163. Accessed December 3, 2017.

71. Indian Immunologicals developing vaccine for chikungunya. The Hindu Business Line; 2012. Available from: https://www. thehindubusinessline.com/companies/Indian-Immunologicalsdeveloping-vaccine-for-chikungunya/article20395594.ece. Accessed October 24, 2017.
Drug Design, Development and Therapy

\section{Publish your work in this journal}

Drug Design, Development and Therapy is an international, peerreviewed open-access journal that spans the spectrum of drug design and development through to clinical applications. Clinical outcomes, patient safety, and programs for the development and effective, safe, and sustained use of medicines are the features of the journal, which

\section{Dovepress}

has also been accepted for indexing on PubMed Central. The manuscript management system is completely online and includes a very quick and fair peer-review system, which is all easy to use. Visit http://www.dovepress.com/testimonials.php to read real quotes from published authors. 